\title{
Observation of an Electron Vortex Beam Created from a Self-Charging Rod
}

\author{
Arthur M. Blackburn ${ }^{1}$
}

${ }^{1 .}$ Hitachi Cambridge Laboratory, Hitachi Europe Ltd., Cambridge, U. K.

Electron vortex beams carry orbital angular momentum and have use in electron microscopy for characterizing the magnetic and chiral properties of materials [1,2]. For these applications it is preferable to have a single isolated vortex beam to simplify the interpretation of scanning probe measurements. A ferromagnetic rod, which creates a spiral like phase shift for electrons passing near its free end when inserted into the beam path, has been shown to produce good quality single electron vortex beams and is well suited to these applications [3]. However, the production of the highest quality ferromagnetic rods requires well controlled lithography (typically around $\pm 10 \mathrm{~nm}$ ), along with process equipment capable of handling ferromagnetic materials. Further, the speed with which the magnetization direction of the ferromagnetic rod can be switched, is limited by the significant losses and response time of any macroscopic electromagnetic coil that is used to magnetize the rod. Thus, there is interest in electrostatic means of creating electron vortex beams, that could allow rapid switching of the handedness of the vortex beam, along with tuning of the orbital angular momentum.

Here, consideration is given to using a dielectric rod with a rectangular cross section which is coated on two contiguous sides with an angled evaporation of a conductive material (Fig. 1(a-b), rather than the previously investigated a ferromagnetic rod [3]. This rod, having a cross-section typically $1 \mu \mathrm{m}$ by 1 $\mu \mathrm{m}$, is then placed in the electron beam with an exposed dielectric face running approximately parallel to the electron beam. This exposed dielectric face consequently charges in the presence of the electron beam and so provides a phase shift to, or equivalently - deflects, electrons passing near this face. To get a sense of the form of this phase shift in the region near the end of a typical rod, a simple electrostatic model is constructed, depicted in Fig 1(c), where integration of the potential, $V$, can be carried out over the beam direction $z$, yielding the map shown in Fig 2(a). It is apparent that the resulting electron phase shift, proportional to $\int V \mathrm{~d} z$, contains a degree of vorticity, though this is combined with astigmatism, a defocus, and other higher order aberrations. Approximately removing the defocus and astigmatism numerically gives the phase shift shown in Fig. 2(b), which is reminiscent of the phase shift resulting from a ferromagnetic rod. Decomposing this phase shift into azimuthal modes [4], reveals that a vortex beam produced from such a rod would comprise mixture of modes (Fig 2(c)). This appears to be of relatively low purity compared to previously investigated ferromagnetic rods.

To test this experimentally a $\sim 100 \mu \mathrm{m} \times \mu \mathrm{m} 1 \times 1 \mu \mathrm{m} \mathrm{SiN}_{\mathrm{x}}$ rod was formed by reactive ion etching, which was subsequently coated with $30 \mathrm{~nm} \mathrm{ZnO}$ by ALD, followed by an angled evaporation of Au to a thickness of $200 \mathrm{~nm}$. The phase shift around the rod end was found by electron holography (Fig. 3(a)). While this approximately agreed with the model, it indicated that one side was charging negatively while the other charged positively, contrary to the anticipation that the conductive side of the rod would remain at ground potential. Placing this rod with its free end centered over a circular electron beam aperture, the resulting electron vortex beam probe could be observed about its focus. With correction of the astigmatism, compared to a plain circular aperture, a vortex-like beam could be observed, which was characterized by a central minimum through focus (Fig. 3(b-d)).

The perceived quality of the produced electron vortex beam was higher than expected, which could 
principally be accounted for following from the observed negative - positive potential occurring across the rod, rather than the anticipated only single side charging, thus giving a more symmetric helical-like phase shift. While the exact mechanisms of this charging are not clear, it appears that this method of generating a vortex beam potentially presents a simple means of producing an electron beam with a degree of vorticity and orbital angular momentum, albeit with a potentially relatively low modal purity. Further, in this demonstration of the principle, no control is given over the sense or magnitude of the charging. However, one can envisage fabricating a rod structure with a controlled isolated electrode, which would allow some control of the resulting orbital angular momentum.

References:

[1] J Verbeeck, H Tian and P Schattschneider, Nature, 467 (2010), p. 301.

[2] A Asenjo-Garcia and F J G de Abajo, Physical Review Letters, 113 (2014), p. 066102.

[3] A. M. Blackburn et al, Microscopy and Microanalysis, S3 (2015), p501.

[4] G. Molina-Terriza et al. Phys. Rev. Lett. 88 (2001) p013601.

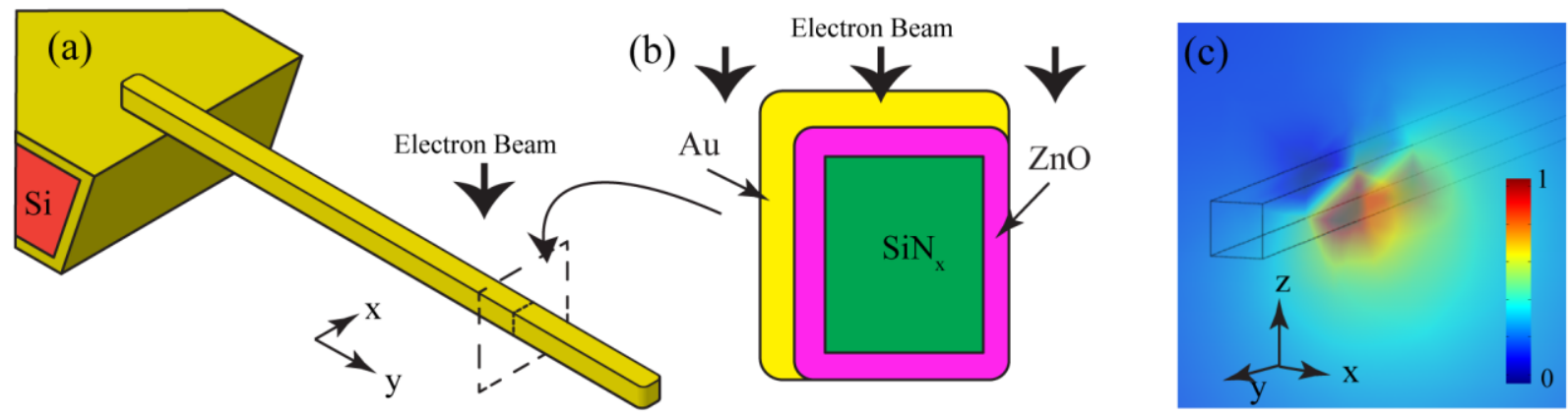

Figure 1. (a) Schematic of the rod used to create the vortex beam, (b) its cross section and (c) representative equipotential map through a section perpendicular to the rod.

(a)

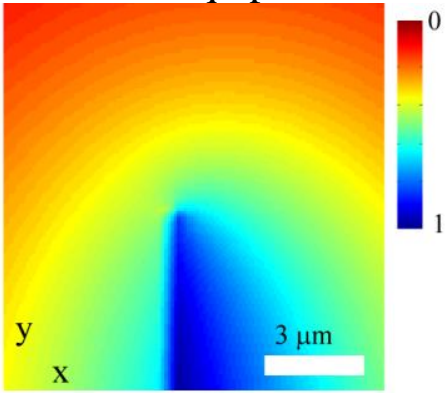

(b)

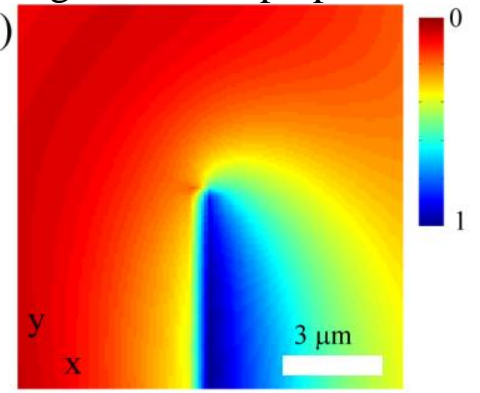

(c)

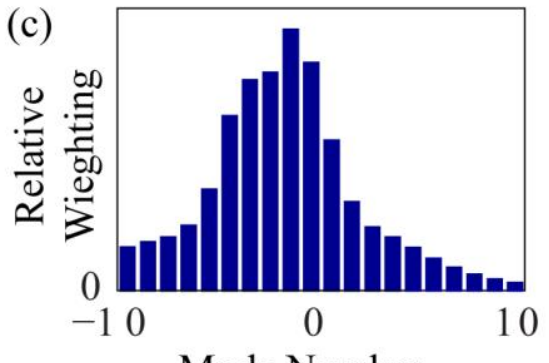

Figure 2. (a) The projected integrated electrostatic potential $\int V \mathrm{~d} z$, is (b) 'flattened' by some astigmatism and defocus aberration correction and then analyzed to give its (c) modal content.
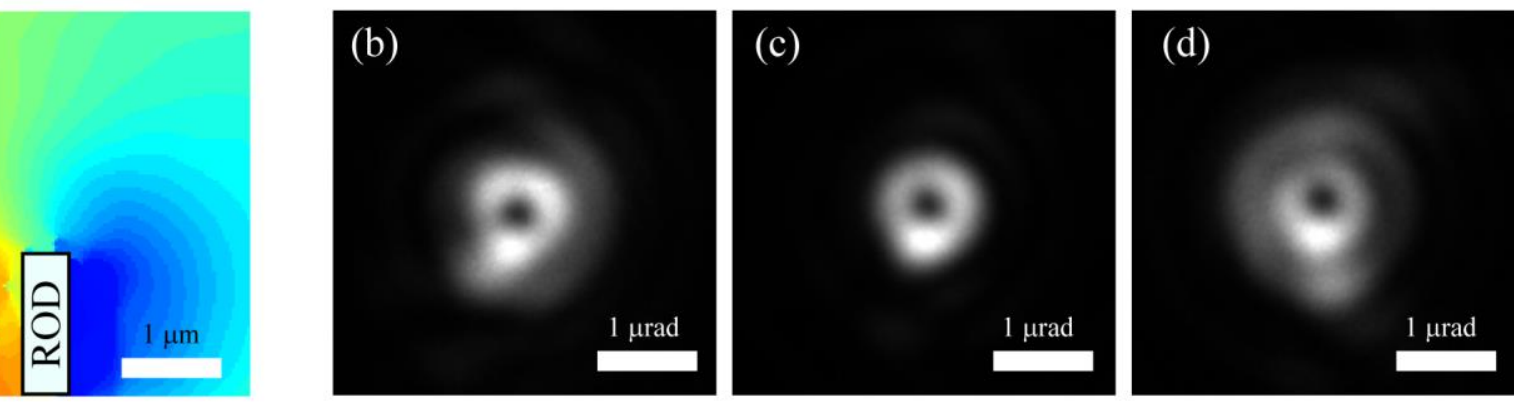

Figure 3. (a) Unwrapped phase from an electron hologram shows the measured phase shift in a typical rod. Observations of the observed $200 \mathrm{keV}$ beam at (b) under, (c) best and (d) over focus all show a central minimum, characteristic of an electron vortex beam. 